\title{
Primary Aorto-Duodenal Fistula as a Late Complication of Radiotherapy: Report of a Case and Review of the Literature
}

\author{
Francesco Puccio Gianpiero Pandolfo Stefano Chiodini \\ Fabrizia Benzi Massimiliano Solazzo \\ Department of Surgery, Hospital of Manerbio, Manerbio, Brescia, Azienda \\ Ospedaliera di Desenzano del Garda (BS), Italy
}

\section{Key Words}

Primary aorto-duodenal fistula - Radiotherapy complication - Gastrointestinal bleeding

\begin{abstract}
Primary aorto-duodenal fistula (PADF) is a rare condition that may result in rapid exsanguination if untreated. PADF due to radiotherapy appears to be extremely rare with only a few cases reported in the medical literature. We report the case of a 61-year-old man who presented with massive gastrointestinal bleeding 25 years after surgery and radiotherapy for seminoma of the testicle and was successfully treated at our institution. We also review the literature on this very uncommon condition. A Medline search was conducted for the period from 1966 to June 2006 to identify case reports of PADF following radiotherapy. Only 7 cases of PADF due to radiotherapy were identified in addition to our own, 4 males and 3 females, aged 40 to 73 years, all treated for various forms of abdominal malignancies. The latency period ranged from 2 weeks to 25 years. None of the aortas were aneurysmatic. One patient died before he could be taken to the operating room. 5 patients underwent surgical repair and 4 survived. 2 patients underwent endovascular treatment but did not survive. PADF may develop up to 25 years after radiotherapy. Diagnosis should be considered when massive upper gastrointestinal bleeding develops in a patient who had previous abdominal radiotherapy, no matter how long before the episode of bleeding. Prompt surgical repair offers a reasonable chance of cure. Endovascular procedures do not appear to be efficacious.
\end{abstract}




\section{Introduction}

Aorto-enteric fistula is a direct communication between the abdominal aorta and the gastrointestinal tract. It is a rare and life-threatening cause of massive upper gastrointestinal bleeding. Primary aorto-enteric fistula is defined as a spontaneous erosion of the aorta into the gastrointestinal tract [1] and must be distinguished from secondary aorto-enteric fistula that may complicate aortic surgery, including endovascular procedures, and is ten times more frequent $[2,3]$. About 250 cases of primary aorto-enteric fistula are reported in the medical literature while its prevalence in large autopsy series has been reported to vary between 0.04 and $0.07 \%[4,5]$. Secondary aorto-enteric fistulas are more common with an incidence varying from 0.4 to $2.4 \%$ of abdominal aortic vascular procedures $[2,3,6-8]$.

Due to its fixed position and close relation to the abdominal aorta, the duodenum is the commonest location of aorto-enteric fistula, usually in its third or fourth part. Common causes of primary aorto-duodenal fistula (PADF) include atherosclerotic aneurysms $[9,10]$ and aortic infections. Salmonella, Klebsiella, mycobacteria, spirochetes and fungi are the microbial species most commonly involved [10-13]. Uncommon causes of PADF include carcinoma of the pancreas [14], biliary stones [15], trauma [16], ingested foreign bodies [17], peptic ulcer disease [18], benign cysts [19] and duodenal diverticular disease [20]. Four cases of idiopathic PADF are reported [21-24], while seven cases of PADF following abdominal radiotherapy for malignancy are described in the medical literature [25-31]. We report a case of PADF developfing 25 years after surgery and radiotherapy and successfully treated at our institution. We discuss the details of the procedure and review the literature pertaining to this uncommon condition.

\section{Case Report}

A 61-year-old man with a recent history of erosive gastritis and duodenal ulcer presented to the emergency department of our hospital with hematemesis, melena and circulatory collapse. His past medical history was remarkable for a left radical orchiectomy and para-aortic lymph node dissection followed by radiotherapy (total dose $45 \mathrm{~Gy}$ ) given 25 years previously for seminoma. An episode of intestinal obstruction due to adhesions was treated surgically a few months after completion of the radiotherapy. An open cholecystectomy for gallstones was carried out in May 2005. One month before admission he had an episode of acute gastroenteritis due to Salmonella enteritidis confirmed by stool cultures. His medications included oral amoxicillin, $1 \mathrm{~g}$ twice daily, and esomeprazole, $40 \mathrm{mg}$ at bedtime.

On examination the patient was alert, oriented and afebrile. His blood pressure was 95/60 $\mathrm{mm} \mathrm{Hg}$ with a pulse rate of $88 / \mathrm{min}$. His skin was pale and clammy. The extremities were cold with feeble distal pulses. Laboratory values included a white blood cell count of $20.96 \times 10^{3} / \mathrm{mm}^{3}$, a hematocrit of $33 \%$, a hemoglobin concentration of $11.5 \mathrm{~g} / \mathrm{dl}$ and a platelet count of $244 \times 10^{3} / \mathrm{mm}^{3}$. Liver function tests, electrolytes, amylase, lipase, blood urea nitrogen and creatinine were normal, as were prothrombin and partial thromboplastin coagulation tests. Digital rectal examination disclosed melena. The patient was resuscitated with a combination of colloids and crystalloids, a nasogastric tube was inserted, revealing copious bright red blood. He was immediately transferred to the endoscopy unit.

Upper gastrointestinal endoscopy showed the presence of active bleeding from the second part of the duodenum. Endoscopic hemostasis with injections of adrenaline 1:10,000 was attempted. A total of $29 \mathrm{ml}$ was injected before apparent control of bleeding was achieved. After the endoscopy an intravenous infusion of omeprazole at high doses was initiated. In spite of these measures, one hour later massive hematemesis recurred with marked hypotension. The patient was therefore transferred to the operating room for emergency laparotomy.

Through a midline laparotomy the gastric antrum and first part of duodenum were opened longitudinally. As no ulcer was found and a large amount of blood was coming from the distal lumen, the incision was extended. The duodenum was mobilized with great difficulty because of extensive and 
dense adhesions due to the previous surgery and radiotherapy resulting in conglomeration of duodenum, aorta and inferior vena cava. Eventually the source of the bleeding was identified as a small-diameter, direct communication between the aorta and the third part of the duodenum. There were no signs of aneurysmatic dilatation, aortitis, or peri-aortic sepsis.

The bleeding was initially controlled by manual compression and the defect in the aortic wall directly repaired with interrupted 2/0 Prolene sutures (fig. 1). At this stage the patient became hemodynamically stable. As the duodenum was widely lacerated and devascularized, we decided to remove it together with the gastric antrum. Only a small portion of the periampullary duodenal wall could be spared. A pedicled omental flap was used to protect the suture in the aortic wall. A Roux-en-Y jejunal loop was used for reconstruction (fig. 2) and a feeding jejunostomy tube inserted. During the procedure the patient received a total of 11 units of packed red blood cells, 4 units of fresh frozen plasma and 4 units of platelet concentrate.

The postoperative course was complicated by the development of a bilio-pancreatic fistula on day seven with an output of about $400 \mathrm{ml} /$ day which was treated with octreotide and total parenteral nutrition. On postoperative day 20 , circulatory collapse due to intraabdominal bleeding suddenly developed. Emergency relaparotomy showed that the bleeding was originating from a large omental vessel and a partial dehiscence of the duodeno-jejunal anastomosis. Hemostasis was carried out and fibrin glue $\left(\right.$ Tissucol ${ }^{\circledR}$ ) was applied to the duodenal remnant in an attempt to control the fistula. A further 5 units of packed red blood cells and 2 units of fresh frozen plasma were transfused.

The subsequent clinical course was complicated by persistent fistula and low-grade sepsis with fever and leukocytosis. A CT scan of the abdomen showed multiple fluid collections which were managed with percutaneous ultrasound-guided drainage and intravenous antibiotics. The patient received a further 5 units of packed red blood cells and 1 unit of fresh frozen plasma. Eventually the fistula output decreased to less than $50 \mathrm{ml} /$ day and oral intake could be resumed. The patient was discharged home two weeks later. A follow-up CT scan 3 months after surgery showed a few small remaining fluid collections and the fistula had closed completely. The patient is alive and well 19 months after discharge and CT scan shows no residual fluid collections.

Histhological examination of the duodenal wall and surrounding tissue at the fistula site showed signs of chronic radiation damage. There was no evidence of chronic ulceration and stains for microorganism were negative. Multiple cultures of blood and stools were negative.

\section{Discussion}

PADF occurs when a degenerative process in the aortic wall erodes directly into the adjacent duodenum. Its incidence is considerably lower than that of secondary aorto-duodenal fistula, which occurs after reconstructive aortic surgery, and less than 200 cases have been reported in the medical literature [32]. Astley Cooper in 1822 first reported a case of PADF caused by the rupture of an aortic aneurysm [33], and Heberer in 1957 successfully treated a patient with PADF by resection of a saccular aneurysm and direct suture and repair of the aorta and duodenum [34].

PADF is associated with an atherosclerotic aortic aneurysm in over $80 \%$ of cases $[1,8-$ 11 ] and aortic wall infection in most of the remaining 20\% [10-13]. Rare causes of PADF include pancreatic cancer, duodenal ulcers, gallstones, duodenal diverticulitis, duodenal trauma, foreign body ingestion and benign cysts. PADF following radiotherapy appears to be extremely rare. Only 7 cases have been reported in the medical literature: 6 cases following external radiotherapy and one case after intraoperative radiotherapy (table 1). In addition, 3 cases of arterio-enteric fistula following surgery for gastric cancer and a combination of intra- and postoperative radiotherapy have been reported [35]. The fistulas originated from the left gastric, the celiac trunk and the superior mesenteric artery, respectively, and are therefore not included in the present review.

Bleeding originated from the third part of the duodenum in 4 cases and from the fourth part in 3 cases. In 3 cases, as in ours, the condition for which the radiotherapy had 
been given was metastatic seminoma of the testis. The time interval between the treatment and the bleeding episode was extremely wide, ranging from 2 weeks to 25 years in our case. In 5 cases extensive surgical dissection in the paraaortic region had been carried out before the radiotherapy was given but, as no aortic reconstruction was made, the fistulas are rightly to be considered primary.

The pathogenesis of postirradiation PADF is unclear, particularly in cases where many years, 25 in our case, have elapsed since the treatment was given. Acute radiation damage to the aortic wall may result in aortic wall necrosis and rupture during or shortly after the treatment $[36,37]$, but the reasons why a communication between aorta and duodenum should develop years or decades after irradiation are far from clear. Radiation damage to the duodenum following high-dose radiation is well described [37] and is explained by its fixed location in the retroperitoneum. It may result in bleeding, ulceration, perforation or fistula formation, often requiring emergency surgical exploration. The histological changes seen in chronic radiation include mucosal and submucosal damage due to obliteration of small vessels, telangiectasis and new vessel formation. These lesions may result in chronic ulcer disease, fibrosis and duodenal stenosis. Chronic inflammation may lead to inflammation and erosion in the surrounding tissues. The role of chronic infection is not clear but is likely to be a contributing factor in the development of a fistula, as well as the pulsatile stress of the underlying aorta onto a weakened duodenal wall.

Although the small bowel is certainly most sensitive to radiation damage, large vessels, including the aorta, may also suffer from radiation injury. Apart from acute rupture during or shortly after radiotherapy, chronic changes are well described, including thrombosis of mural vessels (vasa vasorum) resulting in fibrosis, stenosis and occlusion 10 or more years after treatment and accelerated arteriosclerosis and periarterial fibrosis 20 or more years later [38]. Despite the fact the damage induced by radiotherapy to both the duodenal and aortic wall is well known, the series of events leading to the formation of a fistula between the two structures are still elusive. Most authors seem to favor the hypothesis that a chronic ulcer slowly erodes through the duodenal wall and eventually perforates a weakened and fibrosed aortic wall. In fact an ulcer appeared to be at the origin of the fistula in cases no. 3,5 and $6[15,25,30]$. In the first case a $1.3 \mathrm{~cm}$ ulcer penetrating through the duodenal wall was discovered. The aorta showed a large band of necrosis involving the media and the adventitia while stains for microorganisms were negative. In the second case, occurring 6 years after radiotherapy, a 'radiation ulcer' was found at laparotomy in the third part of the duodenum that had penetrated into the aorta. An ulcer was also present at laparotomy in case no. 7 [25]. In our case the evidence was conflicting. Emergency endoscopy appeared to show a Forrest type 1A bleeding ulcer of the duodenal bulb while at laparotomy no ulcer was seen in that location. As blood appeared to be coming from the distal lumen, the incision was extended along the anterior wall of the duodenum until the fistula was found in the posterior wall of the third portion. We therefore believe that emergency endoscopy resulted in a misdiagnosis that can be well explained if we consider the dramatic setting in which it was carried out. As no evidence of a chronic ulcer was seen in the surgical specimen, the cause of the fistula remains elusive in our case.

\section{Clinical Presentation and Diagnosis}

Clinical presentation of PADF is usually dramatic with massive hematemesis, although the bleeding may be occasionally less severe and cause melena only. In about half the cases a minor 'herald' bleed may precede a major episode hours or even days before a 
catastrophic hemorrhage. Unfortunately the true cause of the bleeding is often misdiagnosed as originating from an ulcer or other condition and therefore adequate measures are not taken to prevent a second, often fatal bleed. The reasons for this repeat bleed are not entirely clear but it is likely due to spasm of the duodenal muscle layer in response to the bleed and hypotension secondary to hypovolemia. In the majority of cases a free interval of $4-24 \mathrm{~h}$ is observed but episodes occurring weeks later are described. In our case a free interval of just one hour occurred, which allowed us to carry out an upper gastrointestinal endoscopy, although this led to a wrong diagnosis. On the other hand, endoscopy is rarely diagnostic in this condition because the orifice of the fistula is generally small and hidden among the folds of the duodenal mucosa. The main purpose of endoscopy is therefore to rule out other causes of bleeding, but it is rarely diagnostic and direct demonstration of an aorto-enteric fistula is a rare event and in most cases the examination is negative or nondiagnostic. On the other hand the misdiagnosis of a bleeding duodenal ulcer may lead to a delay of emergency, life-saving surgical intervention.

In a hemodynamically stable patient $\mathrm{CT}$ can provide very useful information. A gas-containing fluid collection in the paraaortic tissues is an indirect sing of infection surrounding the retro-duodenal aorta. Also, the presence of an aortic aneurysm following an episode of massive upper gastrointestinal bleeding should alert to the possibility of an aorto-enteric fistula.

Angiography is diagnostic in only a minority of cases, as the fistula can be demostrated only in cases of active bleeding who are rarely stable enough to be subjected to this procedure. Further diagnostic evaluation should be pursued only if the clinical condition of the patient permits it. In cases of severe, repeated bleeding the only reasonable measure to be adopted to save the patient's life is surgical intervention. In the largest published series of 118 cases of aorto-duodenal fistula, only $25 \%$ of patients underwent surgical repair and only $60 \%$ of these survived the operation [1].

\section{Treatment}

Emergency surgery by an experienced vascular surgeon provides the only chance of identifying the origin of the fistula and repair it in order to prevent exsanguination and rapid death. Over $75 \%$ of PADFs originate from an aneurysm of the infrarenal descending aorta and in this case the treatment consist in aortic reconstruction using prosthetic material, excision of the fistulous tract and repair of the remaining defect in the duodenal wall. The mortality of this approach lies in the region of about $30 \%$. The notion that local contamination is demonstrated in cultures in about $15 \%$ of cases does not justify the choice of an extraanatomic bypass with its higher morbidity and mortality. This approach should be reserved only to cases with signs of gross local contamination or abscess formation within the periaortic tissues. In the rare cases of radiation-induced PADF a primary surgical repair without use of foreign material appears to be justified. The radiation-damaged portion of the duodenal wall should be excised as a primary repair would certainly result in dehiscence, enteric fistula and abscess formation. A protective 'sleeve' of prosthetic material inserted between the aorta and reconstructed bowel has been used in only one case of the published series and should be considered as a potentially useful adjunct. A pedicled omental graft, as in our case, should also be considered as a protection of the primary repair of the aortic wall. 


\section{Conclusions}

Radiation-induced PADF is a rare and life-threatening cause of massive gastrointestinal hemorrhage. It may develop weeks to many years following radiotherapy. Its etiology has not been fully elucidated but it likely involves chronic inflammatory changes, fibrosis and accelerated atherosclerosis, resulting in weakening of the aortic and duodenal walls. A history of abdominal radiotherapy, no matter how many years before, should be carefully sought in all patients presenting with massive gastrointestinal bleeding.

Endoscopy, CT scan and angiography can be useful in the evaluation of these patients, but are diagnostic in only a minority of cases and a high index of suspicion remains the key to prompt diagnosis and effective treatment. The diagnosis of PADF is generally only confirmed at exploratory laparotomy and all too often is not considered in the differential diagnosis. Once the diagnosis is established or suspected, an urgent laparotomy is mandatory. Direct reconstruction and pedicled omentum plasty, associated with duodenal resection, appear to be the most effective method for closing a PADF.

Our report confirms previous observations that high-energy radiation is a possible etiologic factor in spontaneous PADF, and calls attention to the fact that a fistula may develop even decades after the completion of radiotherapy.

Table 1. Eight cases of primary aorto-duodenal fistula after radiotherapy described between 1972 and 2006

\begin{tabular}{|c|c|c|c|c|c|c|c|c|c|}
\hline $\begin{array}{l}\text { Author, } \\
\text { year, ref. }\end{array}$ & Age & Sex & Basic illness & $\begin{array}{l}\text { Total RT } \\
\text { dose }\end{array}$ & Latency & $\begin{array}{l}\text { Fistula } \\
\text { diagnosed }\end{array}$ & $\begin{array}{l}\text { Duodenal } \\
\text { location }\end{array}$ & Operative procedure & $\begin{array}{l}\text { Sur- } \\
\text { vival }\end{array}$ \\
\hline $\begin{array}{l}\text { Zarembok, } \\
1972[29]\end{array}$ & 40 & M & Hodgkin's disease & $40 \mathrm{~Gy}$ & 2 weeks & autopsy & 4th portion & none & no \\
\hline $\begin{array}{l}\text { Estrada, } \\
1983[28]\end{array}$ & 63 & $\mathrm{~F}$ & $\begin{array}{l}\text { para-aortic lymph } \\
\text { node metastasis } \\
\text { from breast cancer }\end{array}$ & $44 \mathrm{~Gy}$ & 3 months & laparotomy & 4th portion & $\begin{array}{l}\text { direct suture }+ \\
\text { pedicled omentoplasty }\end{array}$ & yes \\
\hline $\begin{array}{l}\text { Nohr, } \\
1990[30]\end{array}$ & 41 & M & seminoma & unknown & 6 years & laparotomy & 3rd portion & direct suture & yes \\
\hline $\begin{array}{l}\text { Fukasawa, } \\
1991[27]\end{array}$ & 67 & $\mathrm{~F}$ & $\begin{array}{l}\text { retroperitoneal } \\
\text { metastases from cancer } \\
\text { of the cervix }\end{array}$ & $55.6 \mathrm{~Gy}$ & 3 months & aortography & 3 th portion & intraaortic balloon & no \\
\hline $\begin{array}{l}\text { Kalman, } \\
1995[26]\end{array}$ & 73 & M & seminoma & unknown & 23 years & laparotomy & 4th portion & direct suture & no \\
\hline $\begin{array}{l}\text { Kitajima, } \\
2000[31]\end{array}$ & 58 & $\mathrm{~F}$ & pancreatic cancer & $\begin{array}{l}25 \mathrm{~Gy} \\
\text { IORT }\end{array}$ & 9 months & aortography & 3rd portion & aortic stenting & no \\
\hline $\begin{array}{l}\text { Drognitz, } \\
2002[25]\end{array}$ & 49 & M & seminoma/teratoma & $50 \mathrm{~Gy}$ & 22 years & laparotomy & 3rd portion & direct suture & yes \\
\hline $\begin{array}{l}\text { Puccio, } \\
2008\end{array}$ & 61 & M & seminoma & $45 \mathrm{~Gy}$ & 25 years & laparotomy & 3rd portion & $\begin{array}{l}\text { direct suture }+ \\
\text { pedicled omentoplasty }\end{array}$ & yes \\
\hline
\end{tabular}


Fig. 1. Intraoperative findings of PADF, with suture of aortic wall.

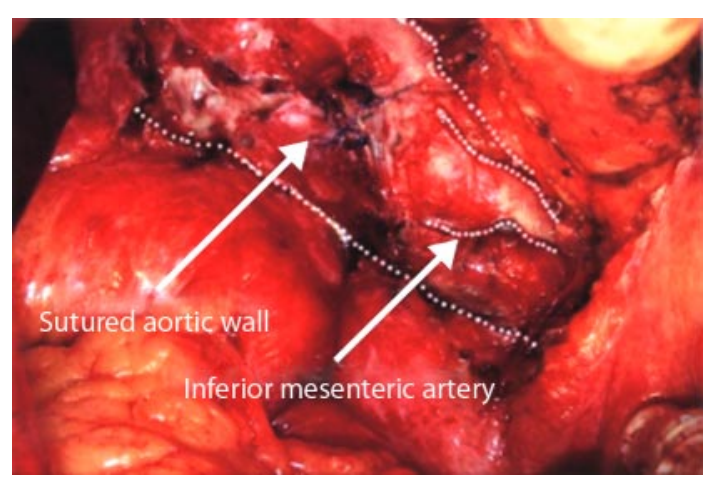

Fig. 2. Schematic drawing of the restored intestinal continuity.

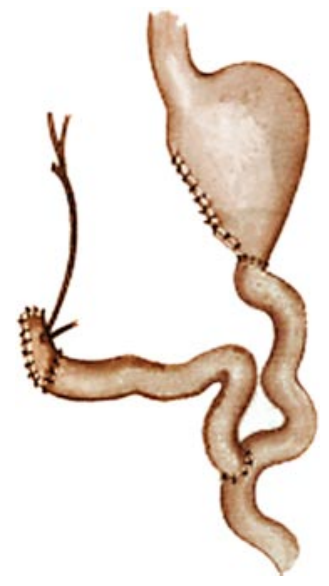




\section{References}

1 Sweeney MS, Gadacz TR: Primary aorto-duodenal fistula: manifestation, diagnosis, and treatment. Surgery 1984;96:492-497.

2 Champion MC, Sullivan SN, Coles JC, Goldbach M, Watson WC: Aortoenteric fistula. Incidence, presentation recognition, and management. Ann Surg 1982;195:314-317.

-3 Flye MW, Thompson WM: Aortic graft-enteric and paraprosthetic-enteric fistulas. Am J Surg 1983;146:183-187.

-4 Kane JM, Meyer KA, Kozoll DD: An anatomical approach to the problem of massive gastrointestinal hemorrhage. AMA Arch Surg 1955;70:570-582.

5 Hirst AE Jr, Affeldt J: Abdominal aortic aneurysm with rupture into the duodenum; a report of eight cases. Gastroenterology 1951;17:504-514.

-6 Bergqvist D, Alm A, Claes G, et al: Secondary aortoenteric fistulas - an analysis of 42 cases. Eur J Vasc Surg 1987;1:11-18.

7 Paaske WP, Hansen HJ: Graft-enteric fistulas and erosions. Surg Gynecol Obstet 1985;161:161-164.

-8 Lemos DW, Raffetto JD, Moore TC, Menzoian JO: Primary aorto-duodenal fistula: a case report and review of the literature. J Vasc Surg 2003;37:686-689.

-9 Reiner MA, Brau SA, Schanzer H: Primary aorto-duodenal fistula. Case presentation and review of the literature. Am J Gastroenterol 1978;70:292-297.

10 Steffes BC, O'Leary JP: Primary aorto-duodenal fistula: a case report and review of the literature. Am Surg 1980;46:121-129.

11 Gad A: Aorto-duodenal fistula revisited. Scand J Gastroenterol Suppl 1989;167:97-100

12 Reckless JP, McColl I, Taylor GW: Aorto-enteric fistulae: an uncommon complication of abdominal aortic aneurysms. Br J Surg 1972;59:458-460.

13 Pagni S, Denatale RW, Sweeney T, McLaughlin C, Ferneini AM: Primary aorto-duodenal fistula secondary to infected abdominal aortic aneurysms: the role of local debridement and extra-anatomic bypass. J Cardiovasc Surg (Torino) 1999;40:31-35.

14 Ghaphery AD, Gupta R, Currie RA: Carcinoma of the head of the pancreas with aorto-duodenal fistula. Am J Surg 1966;111:580-583.

15 Broadbent NR, Taylor DE: Gallstone erosion of the aorta. Aust N Z J Surg 1975;45:207-208.

16 Ferguson MJ, Arden MJ: Gastrointestinal hemorrhage secondary to rupture of aorta. A review of four duodenal and three esophageal cases. Arch Intern Med 1966;117:133-140.

17 Grosfeld JL, Eng K: Right iliac artery-duodenal fistula in infancy: massive hemorrhage due to 'whisk-broom' bristle perforation. Ann Surg 1972;176:761764.

18 Odze RD, Begin LR: Peptic-ulcer-induced aortoenteric fistula. Report of a case and review of the literature. J Clin Gastroenterol 1991;13:682-686.

-19 Sindelar WF, Mason GR: Aortocystoduodenal fistula: rare complication of pancreatic pseudocyst. Arch Surg 1979;114:953-955.

20 Adinolfi MF, Hardin W, Kerstein MD: Aortic erosion by duodenal diverticulum: an unusual aorto-enteric fistula. South Med J 1983;76:1069-1070.

21 Mansel RE, Powley PH: Successful repair of an idiopathic spontaneous aortoduodenal fistula. Br J Surg 1976;63:914-915.

22 Evans DM, Webster JH: Spontaneous aortoduodenal fistula. Br J Surg 1972;59:368-372.

-23 Jaroch MT, Diehl JT, Zippert AM: Primary aortoduodenal fistula without abdominal aortic aneurysm. Cleve Clin Q 1985;52:579-581.

-24 Frizelle FA, Hung NA, Heslop JH, Parry BR: Obscure gastrointestinal bleeding: idiopathic aortoduodenal fistula. J R Coll Surg Edinb 1991;36:331-333.

-25 Drognitz O, Pfeiffenberger J, Schareck W, Adam U, Nizze H, Hopt UT: Primary aorto-duodenal fistula as a late complication of para-aortic radiation therapy. A case report. Chirurg 2002;73:633-637. 
26 Kalman DR, Barnard GF, Massimi GJ, Swanson RS: Primary aorto-duodenal fistula after radiotherapy. Am J Gastroenterol 1995;90:1148-1150.

27 Fukasawa H, Okamoto M, Narushima M, Suzuki A, Udagawa A: A case of aorto-duodenal fistula following radiotherapy of retroperitoneal metastatic disease. Gan To Kagaku Ryoho 1991;18:119-122.

28 Estrada FP, Tachovsky TJ, Orr RM Jr, Boylan JJ, Kram BW: Primary aorto-duodenal fistula following radiotherapy. Surg Gynecol Obstet 1983;156:646-650.

29 Zarembok I, Brace KC: Aorto-duodenal fistula following abdominal irradiation for Hodgkin's disease. A case report. J Can Assoc Radiol 1972;23:267-268.

30 Nohr M, Juul-Jensen KE, Balslev IB, Jelnes R: Primary aorto-enteric fistula: a practicable curable condition? Pathogenetic and clinical aspects. Int Angiol 1990;9:278-281.

31 Kitajima M, Takahashi S, Ueda M, Hoshimoto S, Koizumi J, Matsumoto K, Suzuki H, Takeda A, Kutsuki S, Okita H, Hata J: A case of aortoduodenal fistula occurring after surgery and radiation for pancreatic cancer. Keio J Med 2000;49:35-44.

32 Yao JST, Pierce WH (eds): Arterial Surgery, Management of Challenging Problems. Stamford, Appleton \& Lange, 1996, pp 209-221.

33 Cooper A: Lectures on Principles and Practice of Surgery with Additional Notes and cases By F. Tyrrell, ed 5. Philadelphia, Haswell, Barrington, \& Haswell, 1839.

34 Heberer G: Diagnosis and treatment of aneurysm of the abdominal aorta. Ger Med Mon 1957;2:203-205.

35 De Villa VH, Calvo FA, Bilbao JI, et al: Arterio-digestive fistula: a complication associated with intra-operative and external beam radiotherapy following surgery for gastric cancer. J Surg Oncol 1992;49:52-57.

36 Poon TP, Kanshepolsky J, Tchertkoff V: Rupture of the aorta due to radiation therapy. Report of a case and electron microscopy study. JAMA 1968;205:875878.

37 Fajardo LF, Lee A: Rupture of major vessels after radiation. Cancer 1975;36:904913.

38 Butler MJ, Lane RH, Webster JH: Irradiation injury to large arteries. Br J Surg 1980;67:341-343. 